\title{
A THERMOSENSITIVE LOW MOLECULAR WEIGHT HYDROGEL AS SCAFFOLD FOR TISSUE ENGINEERING
}

Sophia Ziane ${ }^{1,2}$, Silke Schlaubitz ${ }^{1,2}$, Sylvain Miraux ${ }^{4}$, Amit Patwa $^{1,3}$, Charlotte Lalande $^{1,2}$, Ibrahim Bilem $^{1,2}$, Sébastien Lepreux $^{1,2}$, Benoît Rousseau ${ }^{1}$, Jean-François Le Meins ${ }^{5,6}$ Laurent Latxague ${ }^{1,3}$, Philippe Barthélémy ${ }^{1,3}$ and Olivier Chassande ${ }^{1,2, *}$

${ }^{1}$ University of Bordeaux, Bioingénierie Tissulaire, U1026, F-33000 Bordeaux, France

${ }^{2}$ INSERM, Bioingénierie Tissulaire, U1026, F-33000 Bordeaux, France

${ }^{3}$ INSERM, Regulations Naturelles et Artificielles, U869, F-33000 Bordeaux, France

${ }^{4}$ Centre de Résonance Magnétique des Systèmes Biologiques, UMR 5536, Université Bordeaux Segalen - CNRS, Bordeaux, France

${ }^{5}$ University of Bordeaux, LCPO, UMR 5629, F-33600 Pessac, France

${ }^{6} \mathrm{CNRS}, \mathrm{LCPO}, \mathrm{UMR} 5629, \mathrm{~F}-33600$ Pessac, France

\begin{abstract}
Hydrogels that are non-toxic, easy to use, cytocompatible, injectable and degradable are valuable biomaterials for tissue engineering and tissue repair. However, few compounds currently fulfil these requirements. In this study, we describe the biological properties of a new type of thermosensitive hydrogel based on low-molecular weight glycosyl-nucleosyl-fluorinated (GNF) compound. This gel forms within 25 min by self-assembly of monomers as temperature decreases. It degrades slowly in vitro and in vivo. It induces moderate chronic inflammation and is progressively invaded by host cells and vessels, suggesting good integration to the host environment. Although human adult mesenchymal stem cells derived from adipose tissue (ASC) cannot adhere on the gel surface or within a 3D gel scaffold, cell aggregates grow and differentiate normally when entrapped in the GNF-based gel. Moreover, this hydrogel stimulates osteoblast differentiation of ASC in the absence of osteogenic factors. When implanted in mice, gel-entrapped cell aggregates survive for several weeks in contrast with gel-free spheroids. They are maintained in their original site of implantation where they interact with the host tissue and adhere on the extracellular matrix. They can differentiate in situ into alkaline phosphatase positive osteoblasts, which deposit a calcium phosphate-rich matrix. When injected into subcutaneous sites, gel-encapsulated cells show similar biological properties as implanted gel-cells complexes. These data point GNF-based gels as a novel class of hydrogels with original properties, in particular osteogenic potential, susceptible of providing new therapeutic solutions especially for bone tissue engineering applications.
\end{abstract}

Keywords: Low molecular weight gel, supramolecular assemblies, nucleoside amphiphiles, adipose tissue derived stem cells, biocompatibility.

*Address for correspondence:

Olivier Chassande

INSERM - U1026

Université Vioctor Segalen

F-33076 Bordeaux, France

E-mail : olivier.chassande@inserm.fr

\section{Introduction}

Hydrogels have been recognised as having a variety of applications in drug delivery, tissue engineering and regenerative medicine (Drury et al., 2003; Thanos et al., 2008). Beside polymer-based gels, a particular class of hydrogels has more recently emerged that is generated by self-assembly of low molecular weight building blocks, which form supramolecular structures under particular physico-chemical conditions. For example, peptide-based gels have been developed that offer a high versatility in terms of chemical modifications and hence tuneable biological properties (Li et al., 2011; Matson et al., 2011). Thermosensitive hydrogels have the capacity to form gels as temperature increases above the critical solution temperature, designed to be below body temperature (Yu et al., 2008). Such gels offer the advantage that cells or biomolecules can be readily and homogenously incorporated into the gelling matrix. They are also potentially injectable, allowing excellent fitting with the defect or lesion size and shape, potentially avoiding open surgery or minimising invasiveness of biomaterials delivery.

Glycosyl-Nucleoside-amphiphiles are a new class of Low Molecular Weight Gelators (LMWG) possessing a sugar, nucleoside, and lipid covalently linked by triazole bridges. This family is composed of two main categories: the Glycosyl-NucleoLipids (GNLs) (Godeau and Barthélémy, 2009), which possess one or two lipid chains and the Glycosyl-Nucleoside Fluorinated amphiphiles (GNFs) derived from highly hydrophobic fluorocarbon moieties (Godeau et al., 2010). The GNFs used in this study feature three building blocks: a hydrophobic fluorinated carbon backbone, a central thymidine group and a carbohydrate moiety, the fluorinated aliphatic chain and the carbohydrate being linked to the central thymidine (Fig. 1). After solubilisation in an aqueous buffer at high temperature, these compounds have been shown to self-assemble into highly organised supramolecular structures, forming hydrogels at room temperature. Increasing temperature induces gel melting, a property that may have interesting applications such as drug release, if local heating using ultrasounds or thermoprobes can be applied. Considering the similarity in chemical structure and gelling process between GNF and GNLs, the supramolecular organisation of GNF-based hydrogels is 
A

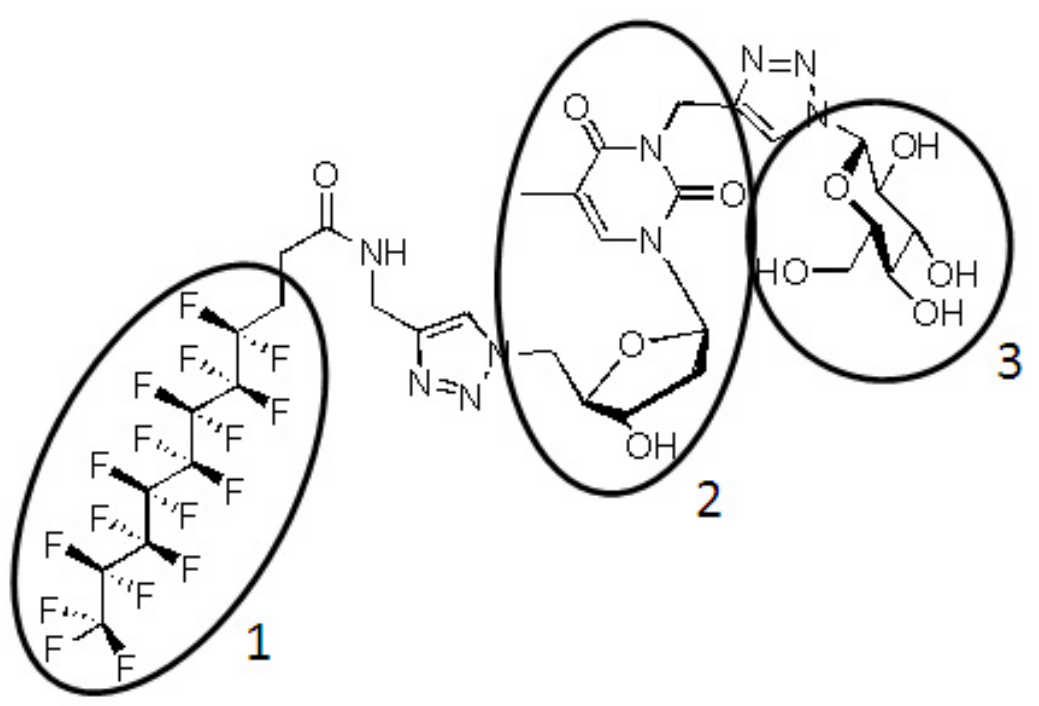

B

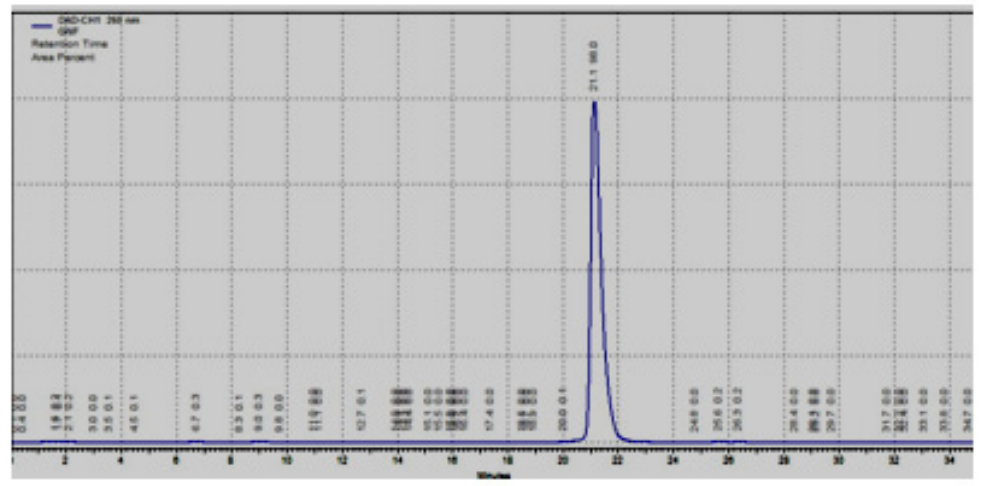

C

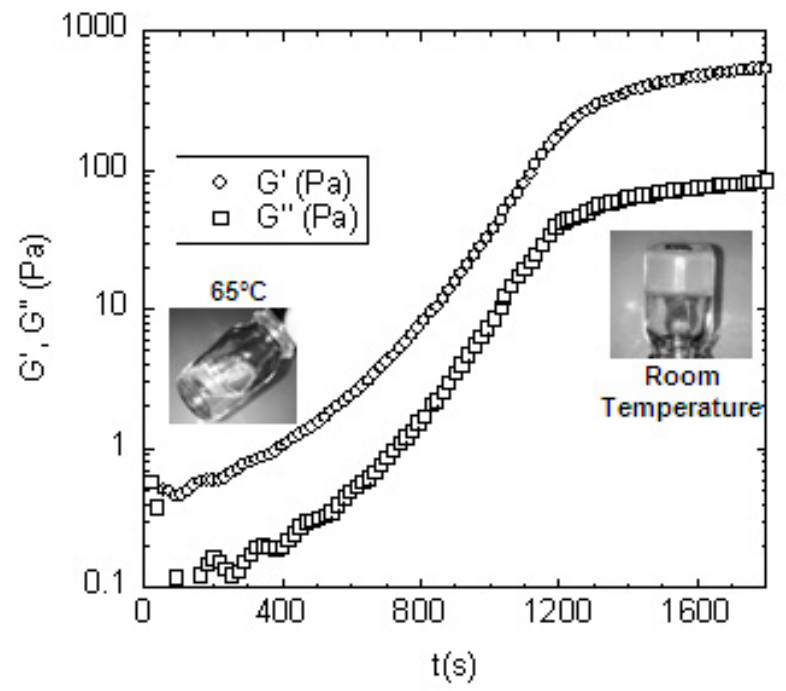

D

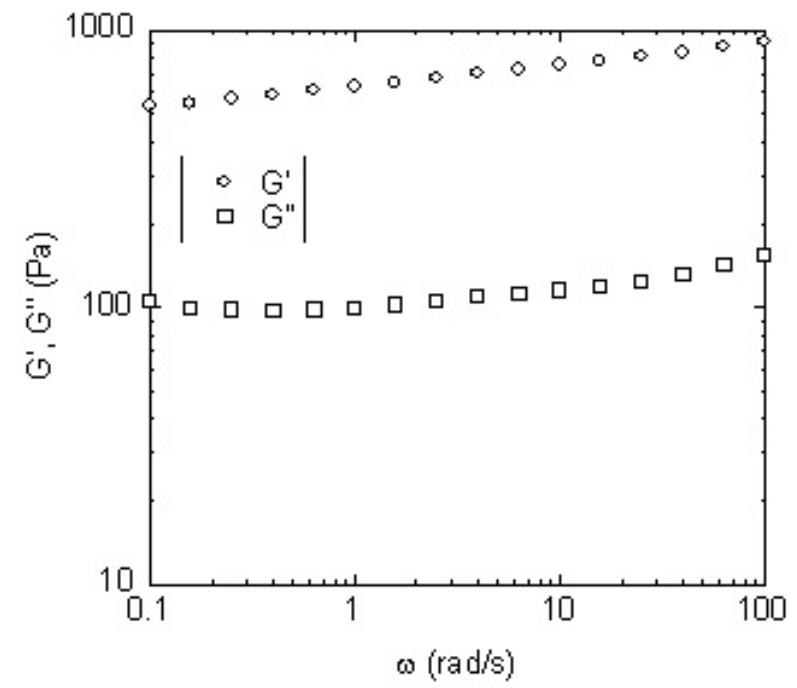

Fig. 1. Rheological properties of a hydrogel formed with pure glycosyl-nucleosyl-fluorinated compound. (A) Developed formula of the GNF compound synthesised by double click chemistry. The fluorinated carbon chain (1) and the glucose moiety (2) are connected to the thymidine (3) by propargyl groups. (B) HPLC profile of the GNF compound following silica gel chromatography. (C) Time course of elastic ( $\left.G^{\prime}\right)$ and viscous (G”) moduli of the GNF-based solution immediately after solubilisation at $65^{\circ} \mathrm{C}$ and transfer of the liquid GNF solution (included picture, left) into the rheometer tank at room temperature. Note that the curves reach a plateau after 1,500 s $(25$ min). At this time, gel is completely formed (included picture, right). (D) Elastic (G') and viscous (G") moduli of GNF-based hydrogels measured at the plateau at different stimulation frequencies. 
likely to resemble the one described for GNL-based gels, consisting of nanofibres with polar sugar heads at the inner and outer surface of nanofibres (Godeau et al., 2009). The presence of sugar on the outer hydrophilic surface offers the advantage of generating a variety of compounds with different functionalities, using different sugar residues or chemically modifying them. Another potential interest of these hydrogels is their capacity to bind nucleic acids, a property that may enable DNA or RNA transfection of cells (Godeau et al., 2009).

Human adipose tissue-derived mesenchymal stem cells (ASC) are multipotent stem cells with the capacity to differentiate into several specialised cells of the mesoderm lineage such as osteoblasts, chondrocytes and adipocytes as well as into cells of the endoderm and ectoderm lineage, according to culture conditions (Zuk et al., 2002). This versatility and the relative facility with which these cells can be obtained make them a promising source of regenerative cells for the treatment of several types of tissue damage (Wilson et al., 2011). ASCs are usually grown as monolayers on plastic culture dishes, where they rapidly proliferate while maintaining their pluripotency. When these cells are grown in non-adherent conditions, they spontaneously form three-dimensional aggregates also called spheroids. These culture conditions modify the properties of ASCs and have been shown to be beneficial for therapeutic applications (Frith et al., 2010).

In this study, we investigated the properties of a GNF-based hydrogel for potential applications in tissue engineering. We evaluated the stability of the gel in vitro and in vivo in subcutaneous position in mouse. We then analysed the interactions of GNF hydrogels with ASCs in vitro. Finally, we analysed the in vivo behaviour of ASChydrogel complexes either after implantation of preformed biomaterials or following injection of liquid gel-cells mixtures.

\section{Materials and Methods}

\section{Synthesis and purification of the GNF compound}

The non-ionic GNF-based amphiphile was synthesised in three steps as described previously (Godeau et al., 2010). Briefly, the three steps synthesis developed to produce the GNF takes advantage of a double-click chemistry approach.

Following a first 'click' reaction, the starting $\mathrm{N}$-propargylated fluorocarbon ( $\mathrm{N}$-propargyl- $2 \mathrm{H}, 2 \mathrm{H}, 3 \mathrm{H}, 3 \mathrm{H}$ perfluoroundecanamide) was reacted with 5'azido-2'deoxythymidine in the presence of $\mathrm{CuSO}_{4}$ and sodium ascorbate in a $\mathrm{THF} / \mathrm{H}_{2} \mathrm{O}$ mixture to afford the expected $2 \mathrm{H}, 2 \mathrm{H}, 3 \mathrm{H}, 3 \mathrm{H}$-perfluoroundecanamide-triazolyl-thymidine intermediate. This fluorocarbon nucleoside intermediate was then treated at room temperature in the presence of potassium carbonate with propargyl bromide to lead to the N-propargylated Thymidine derivative. Finally, the 1 -azido- $\beta$-D-glucopyranosyl moiety was reacted with the N-propargyl Thymidine derivative in the presence of $\mathrm{CuSO}_{4}$ and sodium ascorbate following a second 'click' reaction to provide the expected non-ionic GNF (Fig. 1A). The final product was purified twice by silica gel column chromatography (ethyl acetate/methanol, increasing from $95 / 5$ to $85 / 15$ ), providing pure GNF characterised by NMR. Spectroscopic data agreed with the literature values. The high purity was assessed by a single peak in HPLC analysis (Fig. 1B). HPLC was performed using 2-3 mg GNF dissolved in $1 \mathrm{~mL}$ of Acetonitrile:water (1:4) mixture. The solution was applied to a pre-packed HPLC column 250-4 Nucleosil 120-5 C4 analytical (MASHERYNAGEL). Flow rate was $1 \mathrm{~mL} / \mathrm{min}$ and elution was carried out according to Table 1.

\section{Preparation of GNF hydrogel}

For all in vitro and in vivo assays, GNF-hydrogel was prepared at a concentration of $1.5 \%(\mathrm{w} / \mathrm{v})$. The GNF powder was solubilised in phosphate buffered saline (PBS) at $65^{\circ} \mathrm{C}$, with occasional gentle mixing. For cell culture on hydrogel surface, the GNF solution was poured into plastic culture dish wells. Full gelation was obtained within $20 \mathrm{~min}$ at $37^{\circ} \mathrm{C}$. To entrap cells within the gel, the GNF solution was first cooled down to $37^{\circ} \mathrm{C}$, then immediately mixed with cells and aspired in a $1 \mathrm{~mL}$ syringe. The mixture was deposited in culture dish wells and allowed to gelify. For in vivo implantation, the mixture was allowed to gelify within the syringe, then the gel was expelled by gently pushing the plunger, and sliced as $4 \mathrm{~mm}$ long pieces. For in vivo subcutaneous injections, GNF solution was cooled down at $37^{\circ} \mathrm{C}$, immediately mixed with cells, and the mixture was thoroughly applied to the animal site using a syringe.

\section{Rheological study of the GNF hydrogel}

The viscoelastic properties of the GNF solution were analysed by dynamical mechanical measurement using a stress controlled rheometer (MCR 301, Anton Paar, Courtaboeuf, France) and cone plate geometry (diameter $40 \mathrm{~mm}$, angle $\left.2^{\circ}\right)$. The time dependence of the elastic ( $\left.\mathrm{G}^{\prime}\right)$ and viscous moduli (G”) was analysed $\left(25^{\circ} \mathrm{C}, 1 \mathrm{rad} / \mathrm{s}\right.$, shear strain of $1 \%$ ) immediately after the dissolution process described previously. G' and G' were measured versus frequency (100-0.1 rad/s) at the same temperature after complete gelification.

\section{Adipose tissue derived stem cells (ASCs) culture}

Mesenchymal stem cells were isolated from human adipose tissue. Human subcutaneous fat was obtained from healthy patients aged 20 to 80 years old who underwent hip surgery in Bordeaux Pellegrin CHU (Bordeaux, France). Fat mass was separated from other tissues, washed with sterilised PBS, finely cut and incubated with $0.1 \%$ collagenase (type I, Sigma-Aldrich, St Louis, MO, USA) at $37^{\circ} \mathrm{C}$ with gentle agitation for $1 \mathrm{~h}$. Collagenase activity was stopped with an equal volume of Dulbecco's Modified Eagle's Medium/ F12 (DMEM-F12, Sigma-Aldrich) supplemented with $10 \%$ foetal bovine serum (FBS, Sigma-Aldrich), $100 \mu \mathrm{g} /$ $\mathrm{mL}$ penicillin and $100 \mu \mathrm{g} / \mathrm{mL}$ streptomycin (PS). After centrifugation at $1000 \mathrm{rpm}$ for $10 \mathrm{~min}$ a cell pellet was

Table 1. Elution parameters.

\begin{tabular}{|c|c|c|c|}
\hline $\begin{array}{c}\text { Time } \\
\text { (min.) }\end{array}$ & $\begin{array}{c}\text { \% Solvent A } \\
\text { (Water) }\end{array}$ & $\begin{array}{c}\text { \% Solvent (B) } \\
\text { Acetonitrile }\end{array}$ & $\begin{array}{c}\text { Flow rate } \\
\text { (mL/min) }\end{array}$ \\
\hline 0.0 & 100 & 0 & 1.0 \\
\hline 35.0 & 0 & 100 & 1.0 \\
\hline
\end{tabular}


obtained, suspended in DMEM-F12/10 \% FBS/PS and filtered through a $100 \mu \mathrm{m}$ mesh filter in order to remove debris. The filtrate was centrifuged and cell suspension seeded onto conventional culture flask in controlled atmosphere ( $100 \%$ humidity, $\left.37{ }^{\circ} \mathrm{C}, 5 \% \mathrm{CO}_{2}\right)$. Culture medium was refreshed every three days and cells were passed when confluence reached $80 \%$. For in vitro and in vivo studies ASCs were used at passage 6. ASCs were either seeded on the top of GNF gels at a density of 40,000 cells $/ \mathrm{cm}^{2}$ or mixed with the cooled GNF solution at a concentration of 1.5 million cells $/ \mathrm{mL}$ of gel. To generate ASC spheroid, cells were detached using trypsin/EDTA, recovered and seeded at a density of 2,000 cells per well onto ultra-low-attachment 96-well suspension culture plates (Hydrocell, Thermo Scientific Nunc, Copenhagen, Denmark) and incubated in culture medium in controlled atmosphere. Medium was changed once a week after seeding. To induce ASC differentiation into osteoblasts, cell aggregates were incubated in Iscove's modified Dulbecco's medium (IMDM) supplemented with $10 \%$ FBS, $10^{-7} \mathrm{M}$ dexamethasone, $50 \mu \mathrm{g} / \mathrm{mL}$ ascorbic acid, and $10 \mathrm{mM}$ beta-glycerophosphate for 14 days. Osteoblast differentiation was evaluated by revealing intracellular alkaline phosphatase activity, and calcium deposition in the extracellular matrix. Gel pieces were fixed with 10 $\%$ formalin during $1 \mathrm{~h}$ at room temperature and used for cytochemistry. To stain calcium deposits, samples were incubated in alizarin red (Sigma-Aldrich) (2\% in 0.1 $\mathrm{M}$ acetic acid) for $5 \mathrm{~min}$ and washed five times in PBS. Alkaline phosphatase activity was revealed as previously described (Ackerman et al., 1962). The samples were observed using a Nikon (Tokyo, Japan) Eclipse 80i microscope.

\section{Lentiviral transduction}

The lentiviral vector contained the tdTomato protein gene (Shaner et al., 2004) under the control of the Phosphoglycerate kinase (PGK) promoter. For viral transduction, $2.10^{5}$ freshly trypsinised ASC were mixed with $6.10^{6}$ viral particles $(\mathrm{MOI}=30)$. After $24 \mathrm{~h}$ in culture, virus-containing medium was replaced by fresh medium and cells were allowed to grow. Medium was changed every day for two days. Cells were then amplified and used for in vitro and in vivo assays. Expression of tdTomato was observed under a fluorescent microscope (Zeiss Axiovert 25 CFL microscope; Zeiss, Oberkochen, Germany), with excitation and emission maxima equal to $554 \mathrm{~nm}$ and 581 nm, respectively.

\section{Evaluation of GNF-hydrogel cytocompatibility}

The indirect cytotoxicity of GNF-hydrogel was evaluated by two different assays: cell viability (Neutral Red assay) and cell metabolic activity (MTT assay). GNF hydrogels were incubated in $200 \mu \mathrm{L}$ culture medium for 24,48 or 72 h. Conditioned medium was collected. ASCs were prepared and seeded at a density of 8,000 cells $/ \mathrm{cm}^{2}$ in microtiter plates (Nunc) and cultured in DMEM-F12/10 \% FBS in controlled atmosphere. When cells reached confluency, the culture medium was replaced by gel-conditioned medium. As controls, ASCs were cultured either in standard culture medium (non cytotoxic control) or in presence of phenol at a concentration of $64 \mathrm{~g} / \mathrm{L}$ (cytotoxic control). After the incubation periods, medium was removed and MTT solution or Neutral Red solution was added on cells for 3 $\mathrm{h}$ according the manufacturer's instructions. Colorimetric measurement of formazan and neutral red were carried out on a spectrophotometer at a wavelength of $540 \mathrm{~nm}$. Results were expressed as a percentage of non-cytotoxic control.

\section{Live/dead viability assay}

To evaluate cell viability after 1, 7 or 16 days of culture, aggregated cells were stained using the Live/Dead kit (Molecular Probes) according to the manufacturer's instructions. Spheroids in GNF-gel were incubated for 30 min at $37^{\circ} \mathrm{C}$ in Hank's medium supplemented with 2 $\mu \mathrm{M}$ calcein-AM and $4 \mu \mathrm{M}$ ethidium homodimer (EthD1). The samples were observed using a Zeiss Axiovert 25 CFL microscope.

\section{In vivo implantation of gel-cell complexes}

Bilateral subcutaneous injections or implantations were realised on 10-week-old female NOD-SCID mice (central animal facility of the Université Bordeaux Segalen, Bordeaux, France). TdTomato-tagged ASC spheroids (50 clusters containing 2,000 cells) were either directly injected subcutaneously using a 21-gauge needle, or beforehand mixed with $70 \mu \mathrm{L}$ of GNF hydrogel. Gel-cells mixture was either implanted with forceps, or injected using a 21 -gauge needle in dorsal subcutaneous sites. Five mice were used for each condition and followed for 4 weeks. All procedures and the animal treatment complied with the Principles of Laboratory Animal Care formulated by the National Society for Medical Research. The studies were carried out in accredited animal facilities at the University of Bordeaux Segalen, and were approved by the Animal Research Committee of Bordeaux Segalen University.

\section{In vitro and in vivo imaging of cells}

tdTomato-tagged cells were used in order to monitor the fate of ASCs in a non invasive manner. For the in vitro longitudinal follow-up of cell culture, fluorescence was measured at $540 \mathrm{~nm}$ excitation and $580 \mathrm{~nm}$ emission using an EnVision multilabel plate reader (Perkin Elmer, Courtaboeuf, France). Fluorescence acquisitions were performed after 1, 7, 11, 14 and 18 days of culture. In living mice, fluorescence of cells was measured using the PhotonImager CCDcamera (Biospace ${ }^{\circledR}$, Paris, France). Mice were maintained under anaesthesia by isofurane inhalation. Acquisition time was $10 \mathrm{~s}$ and measurements were performed every week during 1 month. All fluorescence measurements were compensated for the background noise of the GNF hydrogel (negative control).

\section{In vivo imaging of gel by MRI}

Degradation of hydrogel was followed by MRI for 2 months. Experiments were performed on a 9.4T system (Bruker, Ettlingen, Germany) equipped with a $4 \mathrm{~cm}$ gradient system capable of $950 \mathrm{mT} / \mathrm{m}$ maximum strength. Measurements were performed with a birdcage resonator ( $25 \mathrm{~mm}$ diameter and $30 \mathrm{~mm}$ length) tuned to $400.3 \mathrm{MHz}$. In vivo MRI experiments were performed at 1, 30 and 60 days after scaffold implantation. Mice were anaesthetised 

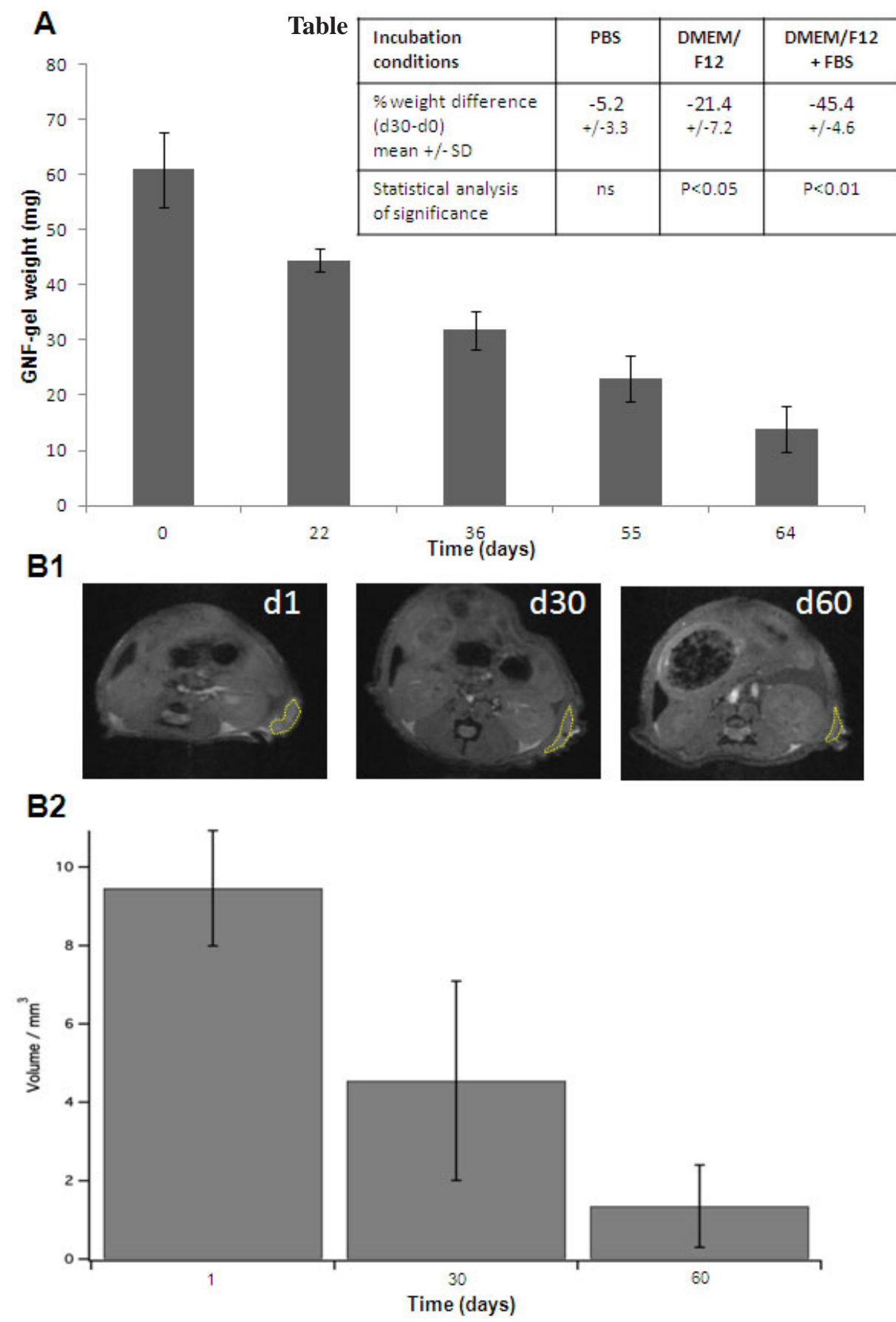

Fig. 2. In vitro (A) and in vivo (B) stability of the GNF hydrogel. (A) Weight of gel pieces after various periods of incubation in DMEM/F12 medium containing $10 \%$ foetal calf serum (graph). 8 gel pieces were weighed at different times after carefully removing excess liquid. In a separate experiment (Table), gel pieces were incubated for 30 days in either phosphate-buffered saline, or in DMEM/F12 without serum or in DMEM/F12 containing 10 $\%$ foetal calf serum. Results are expressed as mean weight \pm standard deviation. (B1) Typical image obtained by MRI of living mice at days 1, 30 and 60. (B2) Volume of subcutaneously implanted gel pieces, determined from MRI images 1, 30 and 60 days after implantation. Results are expressed as mean volume \pm standard deviation.

with isoflurane (1-1.5\% in air) and maintained at a constant respiration rate of $75 \pm 15$ respirations $/ \mathrm{min}$. The animals were positioned prone within the magnet with the liver at the centre of the NMR coil. A 3D TrueFISP imaging (Miraux et al., 2008) with alternating RF phase pulse method and sum of square reconstruction were used as already described (Miraux et al., 2008) (TE/TR: 2.5/5 ms; flip angle: $35^{\circ}$; bandwidth: $271 \mathrm{~Hz} /$ pixel; FOV: 25 x 25 x
20 mm; matrix: 192 x 128 x 96; spatial resolution: 130 x $195 \times 208 \mathrm{~mm}^{3}$ ); number of averages: 3; 8 DeltaPhi values; total acquisition time: $24 \min 35 \mathrm{~s}$ ).

\section{Histological and immunohistochemical analyses}

After 7, 30 or 60 days post-implantation, the mice were sacrificed. Subcutaneous pockets were extracted, fixed in $4 \%$ paraformaldehyde for $4 \mathrm{~h}$ at $4{ }^{\circ} \mathrm{C}$, dehydrated and embedded 

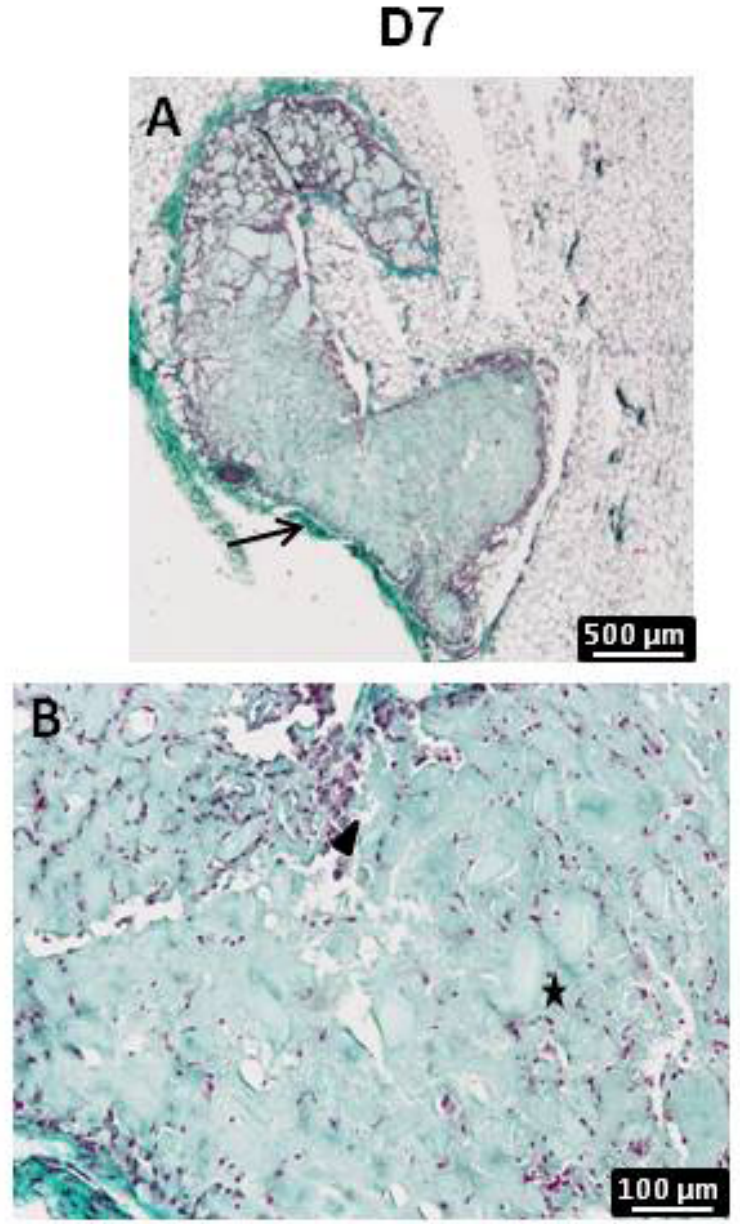

Fig. 3. Histological sections of biopsies 7 days (D7) and 60 days (D60) after subcutaneous implantation of GNF-hydrogel in NOD mice. Sections were stained with Masson's trichrome and examined at x2, x20 and x40 magnification. Stars: gel blocks; Arrowheads: fibroblastoid cells invading the gel; Black arrows: fibrous extracellular matrix; red arrow: blood vessel.
D60
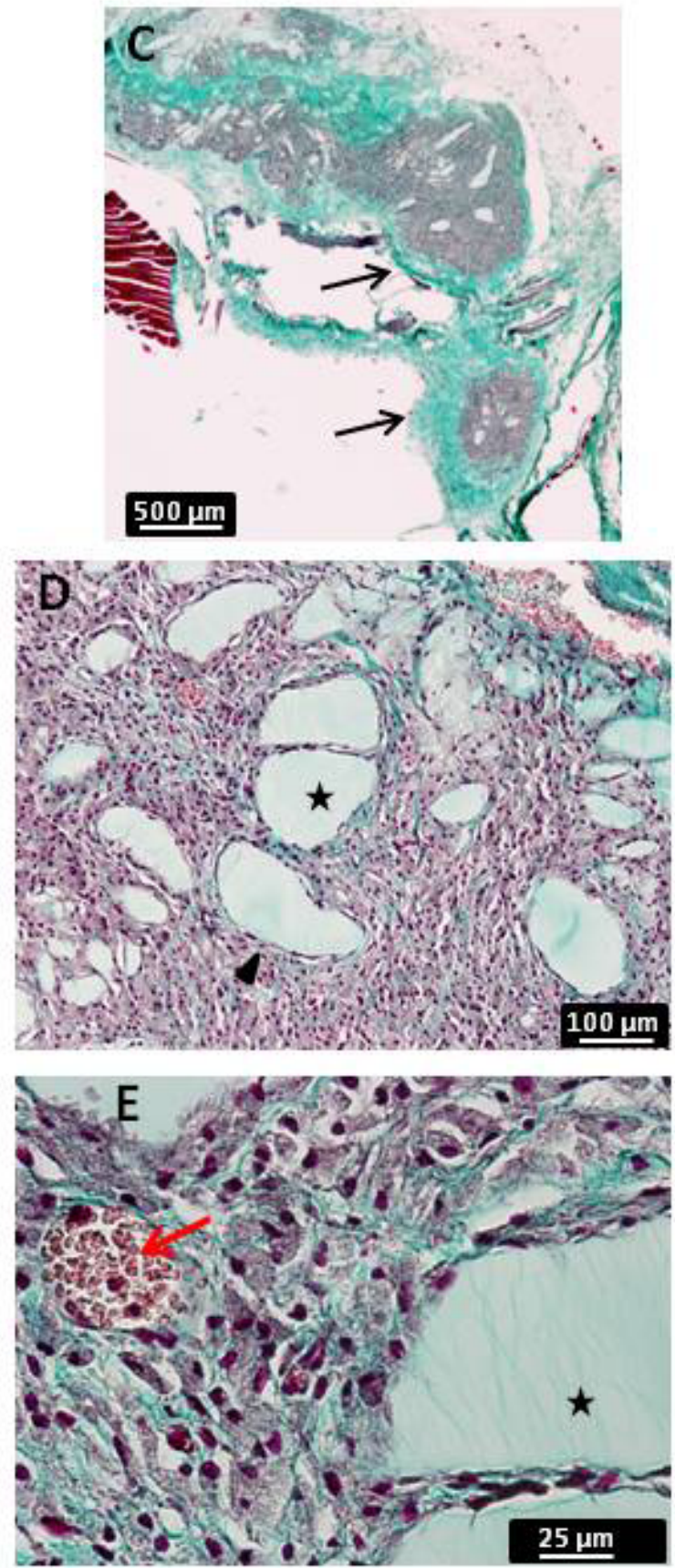

in paraffin. $4 \mu \mathrm{m}$ thick sections were de-paraffinised using toluene, rehydrated in decreasing concentrations of ethanol (100-50\%), washed in distilled water and finally stained with Mayer's hemalum and Erythrosine Masson's trichrome. For immunohistochemistry, antimouse CD68 (1/100) (Abcam, Cambridge, UK) was used as primary antibody. A biotinylated secondary antibody (Invitrogen/Life Technologies, Carlsbad, CA, USA) was used and revealed using Histostain-SP kit (Invitrogen/Life Technologies).

Osteogenic differentiation on paraffin sections was revealed by Alkaline phosphatase (ALP) and Von Kossa staining. For ALP staining, sections were de-paraffinised and then incubated with Fast Blue RR salt and Naphtol AS-MX Phosphate Alkaline solution $0.25 \%$ (Sigma-
Aldrich) during $1 \mathrm{~h}$ at $37^{\circ} \mathrm{C}$ in the dark. Von Kossa positive mineralisation was revealed by incubation of paraffin sections in $2.5 \%$ silver nitrate for $30 \mathrm{~min}$, then in water for $10 \mathrm{~min}$ and finally in $5 \%$ formol sodium carbonate for 3 min. The samples were observed using a Nikon Eclipse $80 \mathrm{i}$ microscope.

\section{Statistical analysis of data}

All statistical analyses were performed using StatEl software (Adscience, Paris, France). For in vitro longitudinal follow-up of TdTomato fluorescence, measurements were performed on 6 different wells per condition. For in vitro assessment of gel weight, 4 pieces were used in each condition. For in vivo MRI imaging of implanted gels, 5 mice were used and followed for 30 days. 

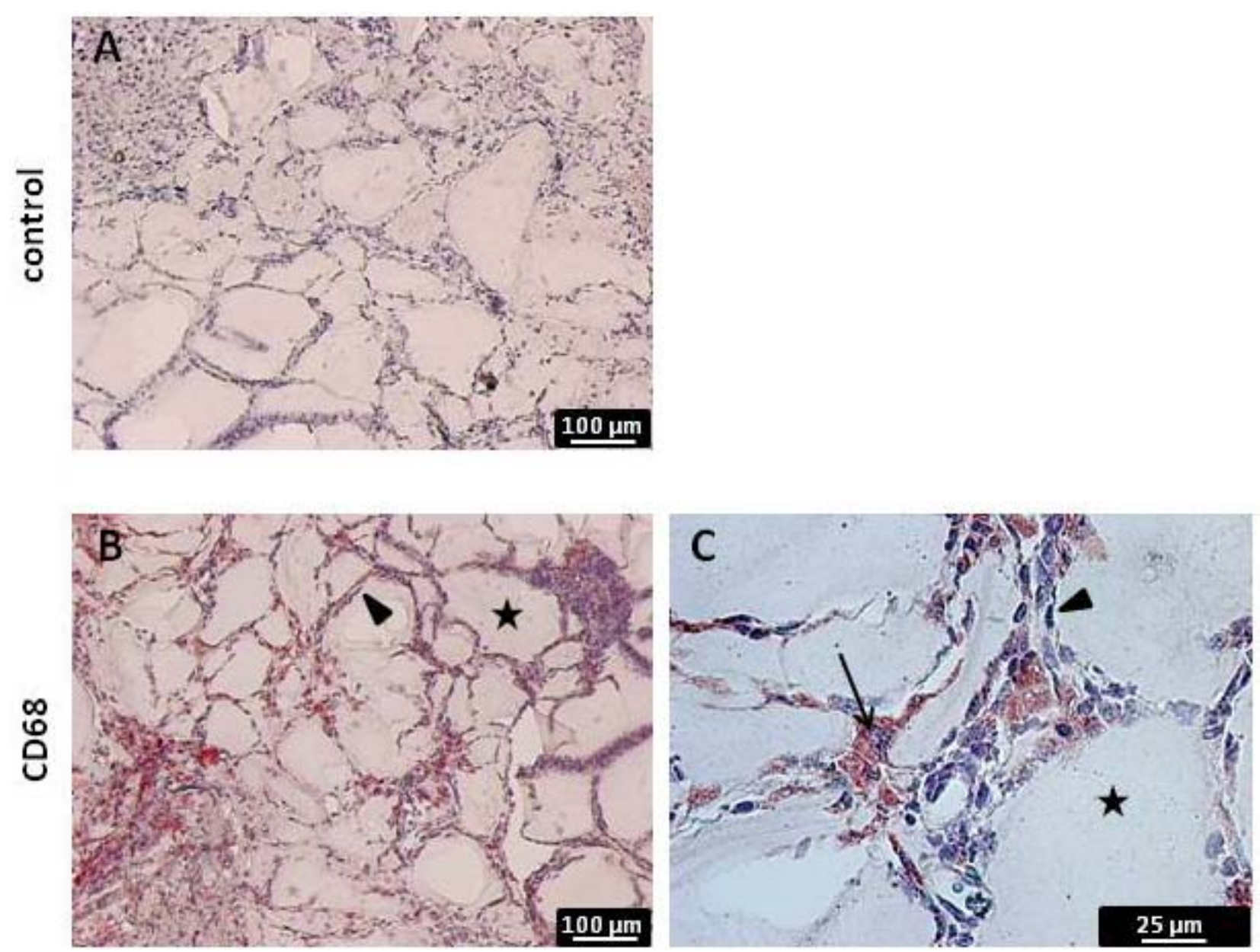

Fig. 4. Histological examination of inflammatory response to GNF gel implantation. Immunohistochemical staining of biopsies 30 days after gel implantation using anti-mouse CD68 antibody. Sections were treated either with (CD68) or without (control) primary antibody. Stars: gel fragments; Arrowheads: fibroblastoid lining cells; arrow: CD68 positive macrophage.

For in vivo measurement of tdTomato fluorescence, 6 mice received Gel-Cells complexes. Assessment of statistical significance of differences observed between time points of kinetics was performed using the paired non-parametric Wilcoxon test.

\section{Results}

\section{Physical properties of the GNF-based hydrogel}

A short preliminary study was performed in order to evaluate the rheological properties of the hydrogel. Fig. $1 \mathrm{C}$ illustrates the time dependence of the elastic and viscous moduli measured just after the preparation process described above. The time passed between aspiration of the warm GNF solution in a syringe, loading into the cone plate geometry, and reaching the first experimental point is estimated to be less than $2 \mathrm{~min}$. It is interesting to note that even at short times $(<200 \mathrm{~s})$ the system presents an elastic modulus higher than the viscous modulus, suggesting that the structuration process is already engaged. Thereafter, both moduli increased before reaching a plateau value at $\sim 30 \mathrm{~min}$, illustrating that the structuration process is almost completed. The final elastic modulus is $\sim 5$ times the viscous modulus. After $30 \mathrm{~min}$, a mechanical spectrum of the GNF sample was acquired. The GNF sample showed a very clear rheological signature of a weak gel in the frequency range investigated, as illustrated in Fig. 1D: the elastic character is dominant, the amplitude of $G^{\prime}$ is around 500-1000 Pa, ( 5 times the value of G") with a slight frequency dependence (Clark and Ross-Murphy, 1987; Almdal et al., 1993; Chronakis et al., 1996).

\section{In vitro and in vivo stability of the GNF hydrogel}

To assess the stability of the GNF hydrogel in vitro, gel pieces were incubated in flasks containing cell culture medium with $10 \%$ serum. Gel blocks were weighed after different periods of incubation. The graph in Fig. 2A shows the linear time course of gel degradation. Extrapolation of the graph shows that half of the initial gel mass was lost after 35 days. To analyse the influence of the incubation medium on gel degradation, gel pieces were incubated for 30 days in phosphate-buffered saline, serum-free culture medium or culture medium containing $10 \%$ foetal calf serum as in the experiment shown in the graph. Interestingly, mass loss was not significant in phosphate-buffered saline (Fig. 2A \& Table), but decreased by $21 \%$ in serum-free culture medium, 
A

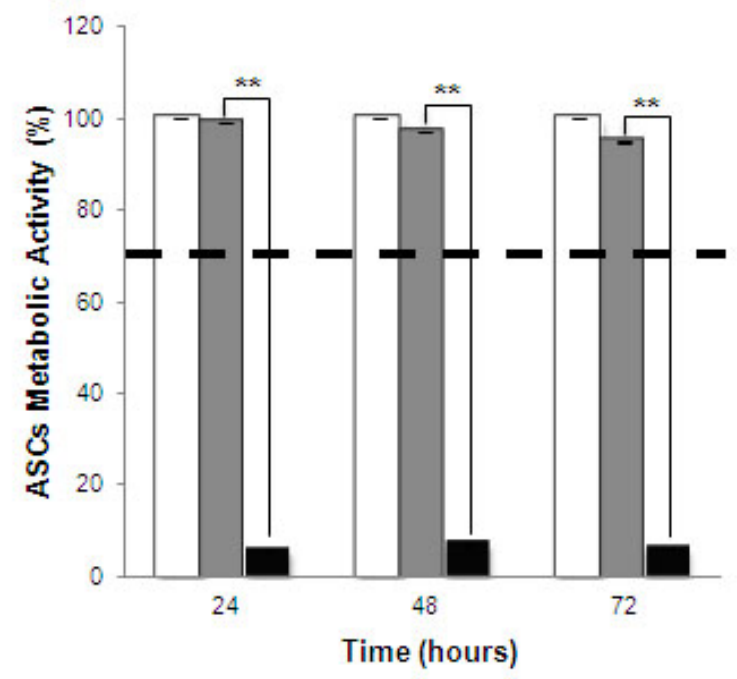

B

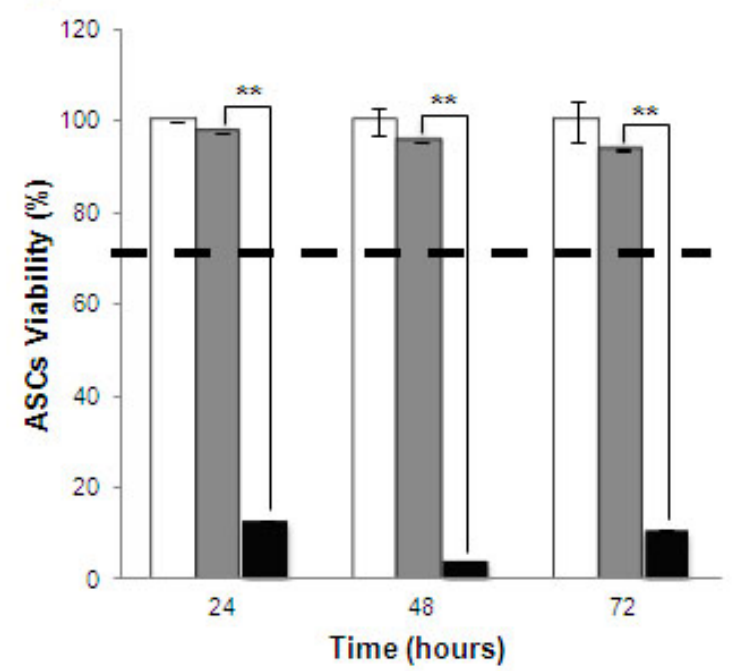

Fig. 5. ASCs metabolic activity (A) and viability (B) assays. ASCs were cultured in medium conditioned with GNFhydrogel for 24, 48 or $72 \mathrm{~h}$. Metabolic activity was evaluated by MTT assay; Viability was evaluated by neutral red uptake. Results (white bars) are expressed as percentage of the values obtained with control samples incubated with normal culture medium (grey bars) and compared to phenol-treated samples (black bars). Statistical significance of differences was assessed by the Mann-Whitney U-test. **: $p<0.01$.

A
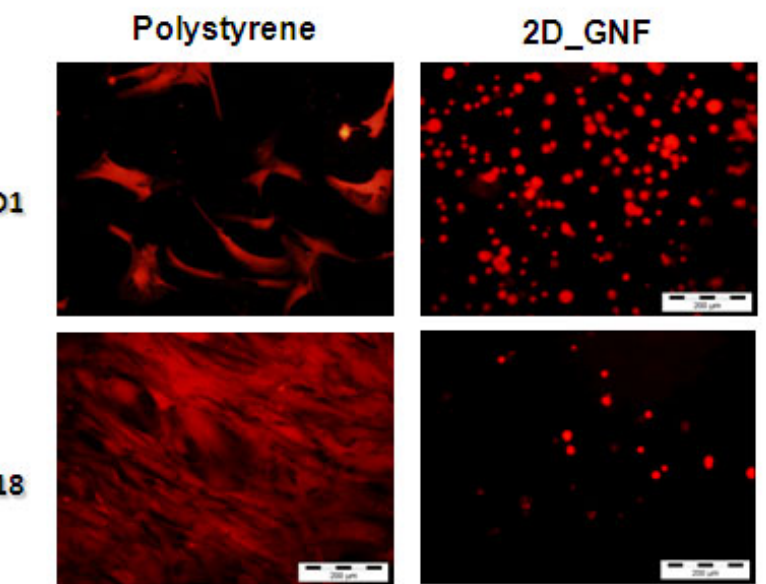

D18

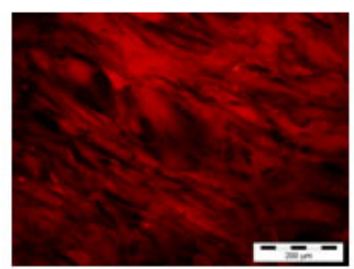

B

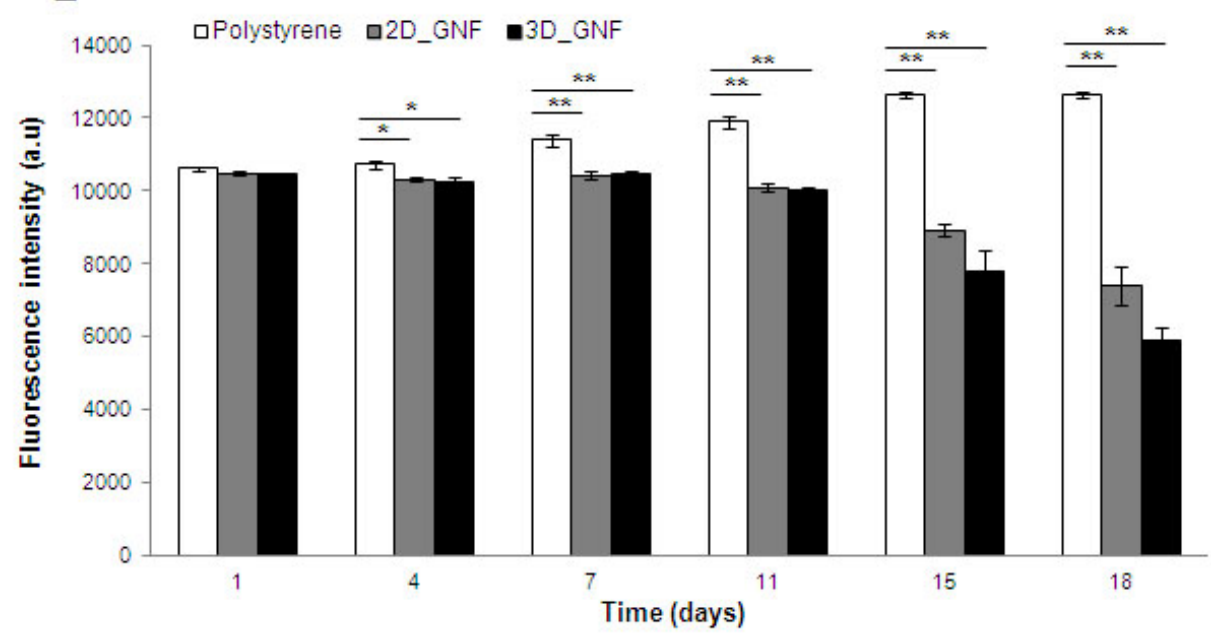

Fig. 6. $2 \mathrm{D}$ and $3 \mathrm{D}$ culture systems using GNF-hydrogel as scaffold. tdTomato tagged ASCs were either seeded onto polystyrene culture dishes (polystyrene, white bars) or on GNF-hydrogel coated wells (2D-GNF, grey bars), or included in GNF-hydrogel (3D-GNF, black bars) and cultured for 18 days. (A) ASCs morphology was observed $24 \mathrm{~h}$ and 18 days after seeding. (B) ASCs growth was followed by measuring the fluorescence of tdTomato protein. Fluorescence is expressed in arbitrary units. Results are expressed as mean \pm standard deviation. Statistical significance of differences was assessed by the Mann-Whitney U-test. *: $p<0.05 ; * *: p<0.01$. 
NS

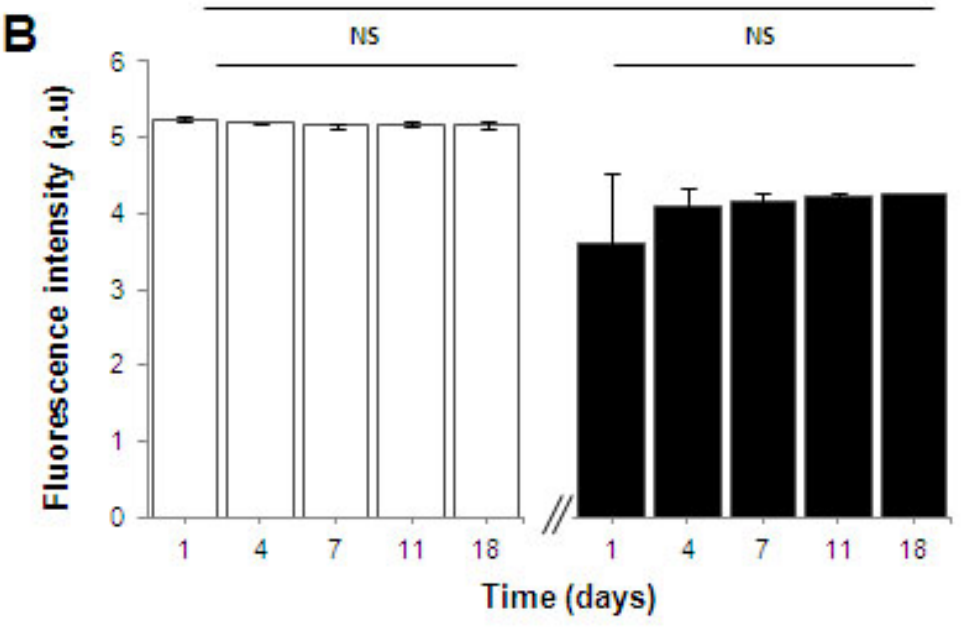

C

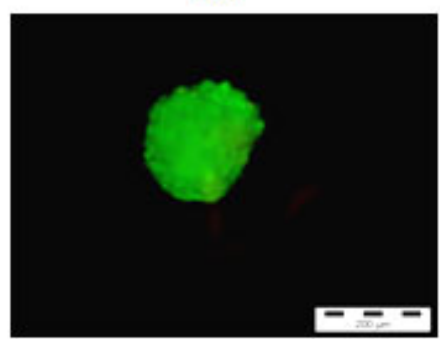

D7

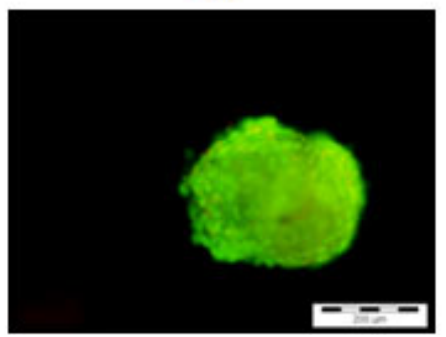

D18

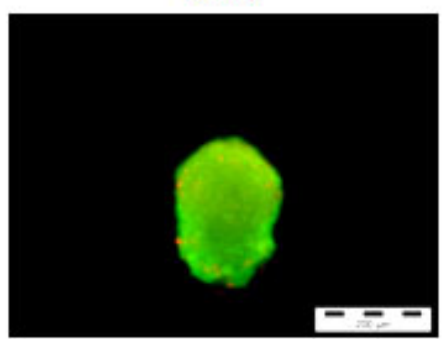

Fig. 7. Culture of tdTomato-expressing ASCs spheroids encapsulated in GNF gel. (A) Merged images of the fluorescences of Td-Tomato and of Hoechst after 21 days of culture. (B) The cell culture growth of free (open squares) or gel-encapsulated (closed squares) clusters was followed by measurement of Td-Tomato protein fluorescence during 18 days. Fluorescence is expressed in arbitrary units. No statistically significant difference was found between time points using Mann-Whitney U-test. (C) Viability of ASCs spheroid entrapped in GNF-gel was analysed by live/ dead assays showing essentially green living cells.

and by $45 \%$ in complete culture medium. These data suggest that gel stability heavily depends on the chemical composition of the medium. Notably, weight decrease correlated with increased fragility of the gel, resulting in fragmentation upon handling. These observations suggest that weight decrease was caused by destruction of the supramolecular structure resulting in progressive gel degradation and fragmentation. Since GNF hydrogels were aimed at in vivo applications, we studied the stability of gel blocks implanted in subcutaneous position in mice. The longitudinal follow-up of implanted gel blocks was achieved by MRI, which allowed the detection of the highly aqueous hydrogel (Fig. 2B1) and the measurement of its volume (Lalande et al., 2011). Gel volume decreased with a linear time course, with half degradation observed around 30 days after implantation (Fig. 2B2). To confirm these data and further analyse the interactions between the GNF-based hydrogel and host tissues, biopsies containing gel pieces were collected either seven days or eight weeks after implantation, and stained with Mayer's hemalum and Erythrosine Masson's trichrome. Fig. 3A,B shows that 7 days after implantation, the gel was still compact, showing very few cracks delineated by scattered host cells. 60 days after implantation (Fig. 3C,D), only few small gel fragments could be observed, that were embedded in fibroblastoid cells. A layer of fibrous extracellular matrix delineated the borders of the implanted gel block. Blood vessels were observed in the fibrous tissue that invaded the gel (Fig. 3E).

We then examined whether GNF-based hydrogels elicited a chronic inflammatory response when implanted in mice. For this purpose, we collected biopsies 30 days after implantation and used antibodies raised against the CD68 antigen, which recognise cell lysosomes, to evaluate the inflammatory response of mouse tissues. Anti-CD68 antibodies revealed the presence of a few macrophages in the vicinity of the gel fragments (Fig. 4B,C), suggesting a moderate chronic inflammation.

\section{Interaction of ASCs with GNF-based hydrogels}

We checked whether the GNF hydrogel could release compounds that could be cytotoxic for ASCs. Culture medium incubated for 24, 48 or $72 \mathrm{~h}$ with GNF-hydrogels showed no significant cytotoxicity when added onto ASCs for $48 \mathrm{~h}$, as assessed by MTT and neutral red assays (Fig. $5 \mathrm{~A}, \mathrm{~B})$. We then analysed the interactions of ASCs with the GNF hydrogel. When ASCs were seeded onto $1.5 \%$ GNF gels, all cells attached to the gel surface after less than $24 \mathrm{~h}$ 

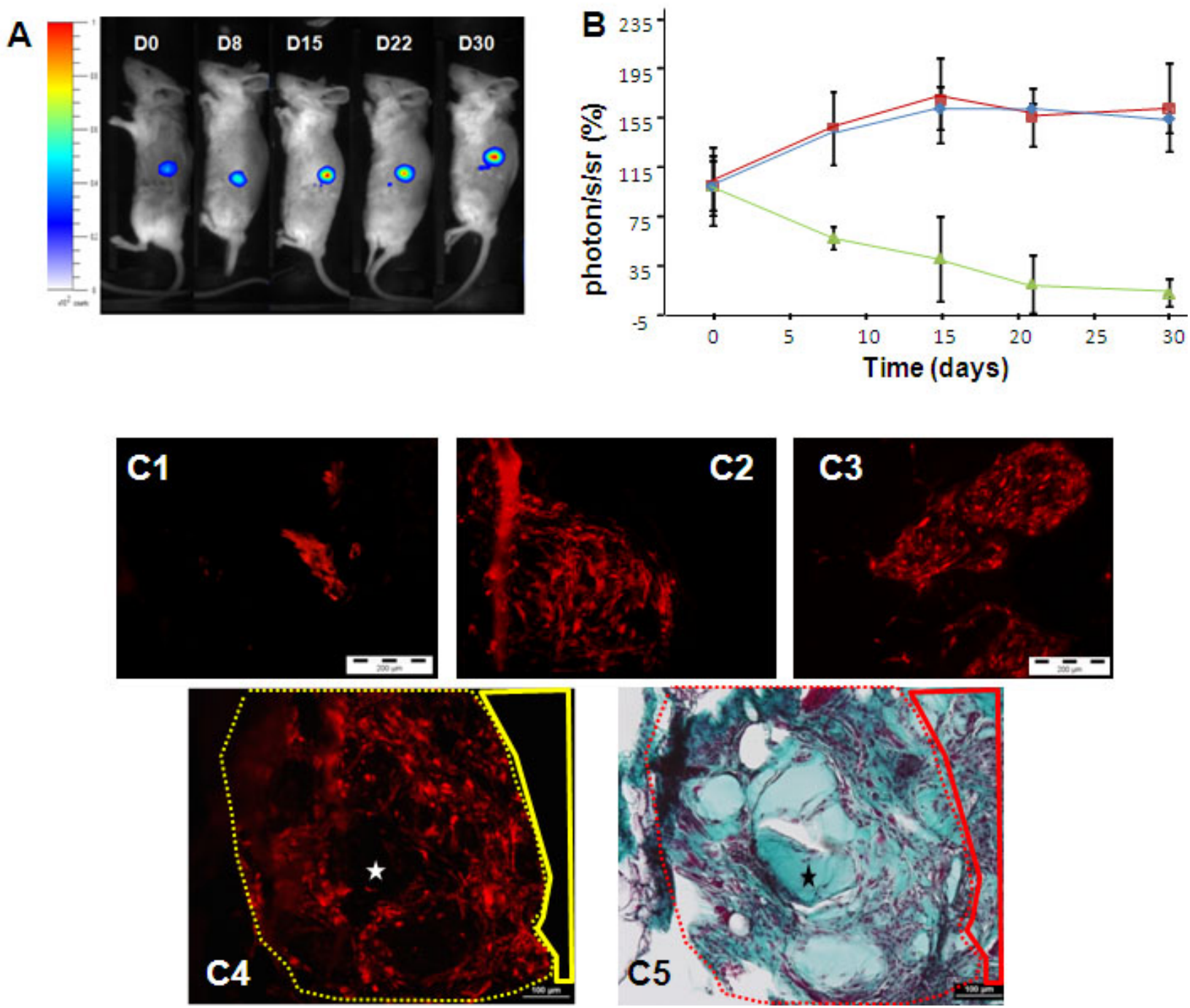

Fig. 8. (A) In vivo imaging of tdTomato fluorescence of ASCs spheroids encapsulated in GNF hydrogels and subcutaneously implanted in NOD-SCID mice. (B) tdTomato fluorescence from either subcutaneously implanted gel-free (green squares) or gel-embedded (red squares) ASCs, or from subcutaneously injected GNF-cell mixture (blue squares). (C) Ex vivo histological observation of tdTomato fluorescence from either subcutaneously implanted gel-free (C1) or gel-embedded (C2) ASCs, or from subcutaneously injected GNF-cell mixture (C3). Biopsies were harvested 30 days after implantation. Observation at high magnification of fluorescent exogenous human ASCs (C4, dotted yellow line) and of endogenous non-fluorescent mouse cells (C5, plain red line). Star indicates gel blocks.

(Fig. 6A, day 1). However, in contrast with cells seeded on polystyrene dishes, the cells did not spread, but retained a round shape. The fluorescence of the stably expressed tdTomato protein increased with time when cells were grown on plastic culture dishes (Fig. 6B). When cells were seeded on the GNF gel, the signal remained stable for one week and then rapidly decreased during the second week in culture medium, suggesting that cell death occurred after one week in these conditions. Very few cells were still present 14 days after seeding (Fig. 6A, day 18).

When cells were entrapped within the gel scaffold, they were uniformly distributed but remained round-shaped (Fig. 6A, 3D_GNF, day 1). Like cells seeded on the gel surface, most of the cells died within 2 weeks of culture (Fig. 6A, 3D_GNF, day 18; Fig. 6B).

ASCs have the property to form aggregates when they are not allowed to adhere to a substrate. Pre-formed ASC aggregates included in the GNF gel matrix retained a spheroid organisation for at least three weeks (Fig. 7A). Cell number within the aggregates, evaluated by the fluorescence of the tdTomato protein, was stable over at least two weeks, either when clusters were cultured as free entities (Fig. 7B, white bars) or when they were included in the GNF hydrogel (Fig. 7B, black bars). Live-dead assays showed the excellent viability of the aggregated cells after two weeks in culture (Fig. 7C).

In vivo assessment of GNF as scaffold for ASC grafting In order to challenge the benefits of GNF hydrogels for cell grafting efficiency, ASCs spheroids expressing the tdTomato protein were implanted into the dorsal subcutaneous region of athymic NOD-SCID mice, in the presence or absence of hydrogel. Cell growth was monitored non-invasively by measuring tdTomato fluorescence emitted from the area of implantation (Fig. $8 \mathrm{~A})$. When cell aggregates were injected without gel, the fluorescence decreased rapidly over time (Fig. 8B). 30 

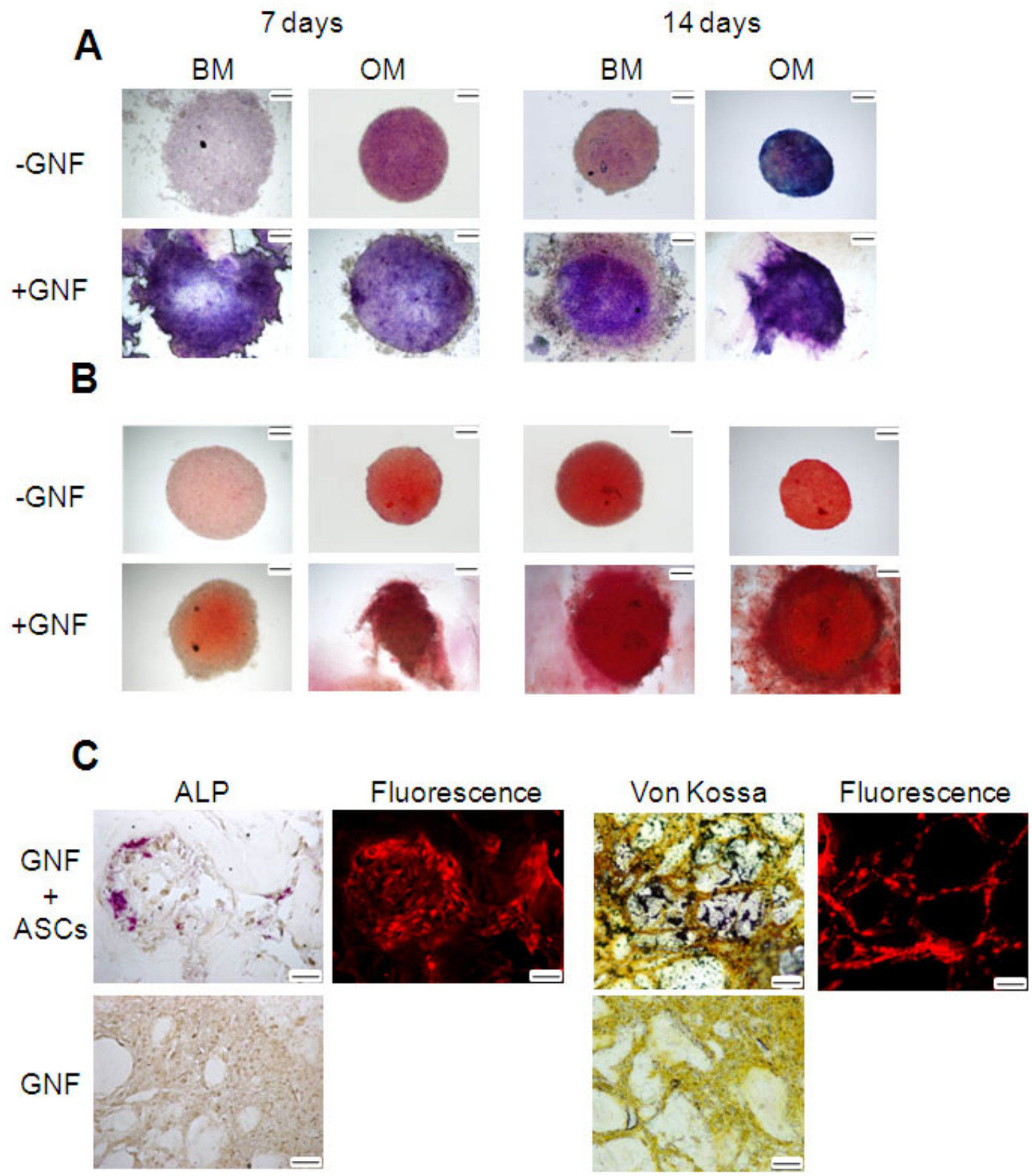

Fig. 9. Effect of GNF hydrogels on the differentiation of ASC spheroids. (A) and (B): In vitro differentiation of ASC spheroids. ASC spheroids are grown for 7 or 14 days, either free (- GNF) or embedded in GNF hydrogels (+ GNF). Cultures are performed in either basal (non osteogenic) medium (BM) or in ostegenic medium (OM). (A) Alkaline phosphatase positive cells and are revealed by blue staining, (B) Calcium-rich extracellular matrix is revealed by alizarin red staining. (C) Identification of osteoblasts in ASC spheroids embedded in GNF hydrogels (GNF + ASCs) and subcutaneously implanted in mice without prior in vitro culture. Biopsies were collected 30 days after implantation. Implanted tdTomato expressing cells show red fluorescence. Alkaline phosphatase cells are stained violet (black arrow) and mineralised matrix appears in black (yellow arrow) after Von-Kossa staining. Gels implanted without human ASCs (GNF) fail to show alkaline phosphatase or Von Kossa positive staining. 
days after implantation, only $18 \%$ of the initial signal was still detected. Histological examination of biopsies 30 days after injection revealed the presence of few scattered fluorescent cells (Fig. 8C1). When these cell aggregates were encapsulated into GNF hydrogels prior to implantation, the fluorescence persisted and even tended to increase with time (Fig. 8B). Biopsies showed a bulk of dense fluorescent cells in biopsies (Fig. 8C2). Human cells were concentrated around the gel pieces (Fig. 8C4) and were not found in the periphery of the implantation site, which contained host cells (Fig. 8C5). Similarly, when cells were mixed with liquid GNF solution and injected, a strong sustained signal was detected at the site of implantation over time (Fig. 8B), and the cells were found as aggregates in biopsies (Fig. 8C3).

\section{GNF hydrogel promotes the differentiation of ASCs into functional osteoblasts in vitro and in vivo}

After showing that the GNF hydrogel enables ASC survival in vitro and in vivo and maintains them in the implantation site, we examined whether it could influence cell differentiation. 7 days after the onset of the culture in basal medium (BM), a significant proportion of alkaline phosphatase (ALP) positive cells were observed in ASC spheroids grown inside GNF hydrogels (+GNF), whereas very few positive cells were found in spheroids grown in the absence of gel (-GNF) (Fig. 9A). Osteogenic medium $(\mathrm{OM})$ enhanced ALP staining in the absence and presence of gel. Extracellular matrix was weakly positive for alizarin red staining only in gel-embedded spheroids, revealing partial mineralisation of the matrix. Osteogenic medium enhanced alizarin red staining in both conditions (Fig. 9B). 14 days after culture onset, gel-associated spheroids contained a large proportion of ALP-positive cells, and exhibited a thick external layer of matrix containing embedded cells. Moreover, this matrix stained strongly positive for alizarin red, suggesting the deposition of calcium phosphate. In contrast, in gel-free spheroids ALP positive cells were fewer and the extracellular matrix was much less extended and alizarin red staining was fainter. Osteogenic medium enhanced ALP and alizarin red staining in gel-free ASC spheroid cultures but did not have any additional effect in the presence of GNF gel. When gel-cells complexes were implanted in mice, ALP, TdTomato positive cells were found within the biopsy (Fig. 9C). Von Kossa staining revealed the presence of calcium phosphate deposits. In contrast, when cell free GNF blocks were implanted, no ALP-positive cells was found and no Von Kossa positive staining was observed.

\section{Discussion}

This study reveals numerous interesting properties of a new type of hydrogel as a vector for cell graft and its potential applications for skeletal tissue engineering. The compound used to form the gel is fully chemically synthesised, avoiding the side effects of natural products in hosts such as allergy and severe inflammation. Gel formation is achieved by cooling the hot GNF solution, avoiding any chemical cross-linking that would require potentially harmful reagents. Moreover, gellification occurs slowly, allowing the safe mixing of cells at $37^{\circ} \mathrm{C}$ before gel formation is completed. This property also offers the possibility to inject the GNF solution and to let it form gel in situ. GNF-based hydrogels show a relatively slow degradation rate, which is similar in vitro and in vivo. This kinetics is compatible with short periods of in vitro cell culture prior to graft. It is also compatible with the requirements of spongious bone tissue engineering, in which degradation of the hydrogel and its replacement by newly formed tissue is desirable (Vinatier et al., 2006). The mechanisms underlying the degradation of the GNF-based gel are unclear. Comparison of gel degradation rates in different media suggests that molecular species within the culture medium affect gel stability. Moreover, the presence of serum significantly accelerates gel degradation, suggesting the participation of proteins in the process. Whether the decrease of gel weight results from a shift in the balance between supramolecular structures and GNF monomers towards soluble monomers, or whether active degradation through enzymes present in the serum requires further investigations. Given the exquisite sensitivity of the gel to the biochemical environment, it is likely that gel stability in vivo will depend on the implantation site. To explore the biological properties of GNF-based hydrogels, we have analysed the biocompatibility of GNF-based hydrogels with cells and tissues. Our studies show that the gel does not release compounds that would be toxic for ASCs, after 3 days of incubation in culture medium. These observations are consistent with previous studies that showed that low concentrations of soluble GNF did not have any cytotoxic effects (Godeau et al., 2009). Thus, GNF-based hydrogels appear cytocompatible. Finally, GNF-based hydrogels produced a moderate chronic inflammation after subcutaneous implantation in mouse. Given the deleterious effects of strong inflammatory reactions for the tolerance and therapeutic efficiency of biomaterials (Nilsson et al., 2010), this property of GNF-based hydrogels appears favourable to tissue engineering applications. Together, these properties point the GNF-based hydrogel as a biocompatible and degradable biomaterial, potentially suitable for tissue engineering applications.

To explore this potential further, the interactions of GNF-based hydrogel with human mesenchymal stem cells isolated from the adipose tissue were studied in different configurations. This scaffold was clearly not compatible with the survival and growth of isolated ASCs, either as two or three dimension cultures. The poor survival of cells is unlikely to be due to the release of toxic compounds from the degrading gel, since our data show the absence of toxicity of gel-conditioned medium and since cell spheroids could survive in these gels. Moreover, as illustrated by the dynamical mechanical analysis, the GNFbased hydrogel presents a typical rheological signature of a weak-gel, suggesting that the poor viability of stem cell is not due to its high stiffness. Instead, we hypothesise that although cells can attach to the gel surface, integrins at the cell surface do not find any substrate within the gel to which they could bind and that would provide the signals 
required for cell survival and differentiation (Prowse et al., 2011). The round shape of the gel-associated cells supports this hypothesis. Although the GNF-based gel is not compatible with the growth of isolated cells, our data show that it supports the survival of ASC aggregates. Cell aggregates have several potential advantages for tissue engineering. ASCs have been shown to differentiate into several cell types more efficiently than adherent cultures on plastic dishes (Frith et al., 2010). ASC aggregates have also been reported to exhibit anti-inflammatory properties (Bartosh et al., 2010). Of note, our data show that ASCs embedded in GNF-based hydrogels are engaged into osteoblast differentiation in basal medium. This effect may be caused by degradation products of the gel, which could induce cell differentiation. Alternatively, it may be due to the microenvironment created by the gel, which results in cell confinement and may favour the local concentration of osteogenic cell-secreted cytokines.

Since GNF-based hydrogel proved to be a good carrier for cell aggregates, we examined its potential benefits for their in vivo implantation. Whereas in the absence of gel, cells progressively disappeared from the implantation site, encapsulated spheroids survived for at least several weeks. It is not possible to determine whether loss of unprotected cells resulted from cell death or cell migration towards other sites. Therefore, the mechanisms by which the gel prevents cell loss remain unclear. Remarkably, a few weeks after implantation, exogenous cells are not only alive and concentrated at the implantation site, but they also exhibit typical fibroblastoid morphology, suggesting that they have lost the spheroid organisation, and that they deposit extracellular matrix and adhere to it. Moreover, the presence of calcium phosphate in the vicinity of gel-associated ASCs and the identification of alkaline positive cells around the gel pieces reveal that the GNF-based hydrogel promotes osteoblast differentiation in vivo, without prior treatment with osteogenic factors. Our data also show that host cells can colonise the gel as it degrades, suggesting that they can interact with implanted exogenous cells. Finally, they show that blood vessels invade the gel fragments, providing a vascularised environment to the exogenous cells. Another advantage of GNF- hydrogels is easy injection of entrapped cells, which remain concentrated at the implantation site and survive for weeks. Their behaviour is very similar to the one of cells incorporated to the hydrogel prior to implantation, suggesting that in situ gel formation is equivalent to prior in vitro gelling in terms of biological properties.

In summary, our investigations point to GNF-based gels as a novel class of hydrogels with a panel of original biological properties which make them promising agents for bone tissue engineering. In particular, the possibility to inject gel-cell complexes, the capacity of encapsulated cells to differentiate into osteoblasts without osteogenic factors point to this hydrogel as a promising tool for the regeneration of spongious bone. Moreover, due to the modularity of the chemical structure of GNF compounds, it will be possible to design and synthesise several new molecules differing either by their sugar, nucleoside or aliphatic chain moiety and displaying a variety of biological properties.

\section{Acknowledgements}

This work was supported by the French National Agency (ANR) in the frame of its Programme Blanc (project GelCells, appel à projets Blanc SIMI 7-2010).

\section{References}

Ackerman GA (1962) Substituted naphthol AS phosphate derivatives for the localization of leukocyte alkaline phosphatase activity. Lab Invest 11: 563-567.

Almdal K, Dyre J, Hvidt S, Kramer O (1993) Towards a phenomenological definition of the term 'gel'. Polym Gels Networks 1: 5-17.

Bartosh TJ, Ylöstalo JH, Mohammadipoor A, Bazhanov N, Coble K, Claypool K, Lee RH, Choi H, Prockop DJ (2010) Aggregation of human mesenchymal stromal cells (MSCs) into 3D spheroids enhances their antiinflammatory properties. Proc Natl Acad Sci USA 107: 13724-13729.

Chronakis IS, Piculell L, Borgström (1996) Rheology of kappa-carrageenan in mixtures of sodium and cesium iodide: two types of gels. J Carbohydr Polym 31: 215-225

Clark AH, Ross-Murphy SB (1987) Structural and mechanical properties of biopolymer gels. Adv Polym Sci 83: $57-192$

Drury JL, Mooney DJ (2003) Hydrogels for tissue engineering: scaffold design variables and applications. Biomaterials 24: 4337-4351.

Frith JE, Thomson B, Genever PG (2010) Dynamic three-dimensional culture methods enhance mesenchymal stem cell properties and increase therapeutic potential. Tissue Eng Part C Methods 16: 735-749.

Godeau G, Barthélémy P (2009) Glycosyl-nucleoside lipids as low-molecular-weight gelators. Langmuir 25: 8447-8450

Godeau G, Bernard J, Staedel C, Barthélémy P (2009) Glycosyl-nucleoside-lipid based supramolecular assembly as a nanostructured material with nucleic acid delivery capabilities. Chem Commun (Camb) 14: 5127-5129.

Godeau G, Brun C, Arnion H, Staedel C, Barthélémy P (2010) Glycosyl-nucleoside-fluorinated based amphiphiles as components of nanostructured hydrogels. Tetrahedron Lett 51: 1012-1015

Lalande C, Miraux S, Derkaoui SM, Mornet S, Bareille R, Fricain JC, Franconi JM, Le Visage C, Letourneur D, Amédée J, Bouzier-Sore AK (2011) Magnetic resonance imaging tracking of human adipose derived stromal cells within three-dimensional scaffolds for bone tissue engineering. Eur Cell Mater 21: 341-354.

Li X, Yi Kuang, Shi J, Gao Y, Lin H-C, Xu B (2011) Multifunctional, biocompatible supramolecular hydrogelators consist only of nucleobase, amino acid, and glycoside. J Am Chem Soc 133: 17513-17518.

Matson JB, Stupp SI (2012) Self-assembling peptide scaffolds for regenerative medicine. Chem Commun (Camb) 48: 26-33.

Miraux S, Massot P, Ribot EJ, Franconi J-M, Thiaudiere E (2008) 3D TrueFISP imaging of mouse brain at 4.7T and 9.4T. J Magn Reson Imaging 28: 497-503. 
Nilsson B, Korsgren O, Lambris JD, Ekdahl KN (2010) Can cells and biomaterials in therapeutic medicine be shielded from innate immune recognition? Trends Immunol 31: 32-38.

Prowse ABJ, Chong F, Gray PP, Munro TP (2011) Stem cell integrins: implications for ex-vivo culture and cellular therapies. Stem Cell Res 6: 1-12.

Shaner NC, Campbell RE, Steinbach PA, Giepmans BNG, Palmer AE, Tsien RY (2004) Improved monomeric red, orange and yellow fluorescent proteins derived from Discosoma sp. red fluorescent protein. Nature Biotechnology 22: 1567-1572

Thanos CG, Emerich DF (2008) On the use of hydrogels in cell encapsulation and tissue engineering system. Recent Pat Drug Deliv Formul 2: 19-24.

Vinatier C, Guicheux J, Daculsi G, Layrolle P, Weiss $P$ (2006) Cartilage and bone tissue engineering using hydrogels. Biomed Mater Eng 16: 107-113.

Wilson A, Butler PE, Seifalian AM (2011) Adiposederived stem cells for clinical applications: a review. Cell Prolif 44: 86-98.

Yu L, Ding J (2008) Injectable hydrogels as unique biomedical materials. Chem Soc Rev 37: 1473-1481.

Zuk PA, Zhu M, Ashjian P, De Ugarte DA, Huang JI, Mizuno H, Alfonso ZC, Fraser JK, Benhaim P, Hedrick MH (2002) Human adipose tissue is a source of multipotent stem cells. Mol Biol Cell 13: 4279-4295.

\section{Discussion with Reviewers}

Reviewer I: In the cell attachment experiments, normally cells will attach to the biomaterial surface by interacting with binding proteins from the serum, such as serum soluble fibronectin and others. If the serum is helping the hydrogel to degrade, it makes sense that the surface is unstable and therefore loaded cells will have trouble in binding to it. Please, make comments on this aspect. The same can be said when cells are encapsulated, where low cell-surface interaction is really observed. In this case, cells within the spheroid interact with each other and not with the matrix.

Authors: This is an interesting hypothesis. However, we think that the very low rate of degradation cannot account for the low interaction of cells with the gel. Moreover, we do observe cell attachment, but not cell adhesion and spreading, suggesting that interaction occurs, but not through integrins, obviating cell survival signals.

Reviewer II: The authors report a moderate chronic inflammation after subcutaneous implantation in mouse, which is a very good outcome, but when targeting bone repair, the low molecular weight hydrogel will be located in a damaged organ, close to bone marrow, which can then elicit a stronger inflammation/foreign body reaction. Could the authors comment and suggest a possibility to decrease the potential foreign body reaction of self-assembling hydrogels?

Authors: We have performed preliminary experiments in which we injected the GNF-based hydrogel into a epimetaphyseal femur defect in rat. Neither MRI images nor histological analysis of longitudinal sections revealed significant inflammation. In parallel with the current studies focusing on human stem cells-hydrogel interactions, we are developing investigations aimed at encapsulating different molecules in nanoparticles, which could themselves be entrapped in gels for controlled drug delivery. However, these studies have just been initiated.

Reviewer II: The authors suggested that GNF-based gel is not compatible with the growth of isolated cells. Did the authors tried to encapsulate chondrocytes or nucleus pulposus cells in their hydrogels? The GNF hydrogels may be more adequate for cartilage or nucleus pulposus tissue engineering. It would be interesting to have the comment of the authors on this aspect.

Authors: Our data clearly show that the GNF hydrogel does not allow the growth of isolated mesenchymal stem cells. We are currently designing composite hydrogels associating the GNF-based gel with other matrix proteins such as collagen. The aim is to obtain gels that would allow cell adhesion (through the non-GNF matrix) and osteoblast differentiation (through the effect of the GNF gel, as shown in the study described in this paper). Besides, we are starting studies with chondrocytes from articular cartilage. We expect the GNF hydrogel to be compatible with the growth of chondrocytes. Indeed these cells have been show to grow and differentiate in a variety of hydrogels without the need for adhesion.

Reviewer II: It is very interesting that the GNF can induce "osteogenic" like behaviour of cells in pellet in a basal medium. It would be interesting to have the comments of the authors and how the mechanism of such phenomena could deciphered. Would it be possible to know if this is a clustering effect or an effect of the GNF single molecules? Authors: Our preliminary studies suggest that the single GNF molecules do not have any positive effect of osteoblastic differentiation of mesenchymal stem cells. These experiments have been performed by adding increasing concentrations of GNF (however at lower concentrations than those used to allow gel formation). Hence, we infer that the mechanisms accounting for the commitment to osteoblast are more related to the clustering effect. Local accumulation of growth factors is one possible mechanism. 